\title{
Angiodysplasia of the small bowel; a 'brilliant' technique for localizing the quiescent lesion
}

\author{
S.D. Scott and G.T. Royle \\ University Surgical Unit, F Level, Centre Block, Southampton General Hospital, Tremona Road, Southampton \\ SO1 6HU, UK.
}

\begin{abstract}
Summary: Intraoperative transluminal endoscopic illumination is of benefit to locate angiodysplasias of the small bowel at laparotomy in the quiescent non-bleeding phase.
\end{abstract}

\section{Introduction}

Angiodysplasia of the small bowel is uncommon, ${ }^{1,2}$ but probably remains frequently undiagnosed. The condition is commonest in the ascending colon ${ }^{1,3}$ where it may occur in $25 \%$ of people over 60 years of age. ${ }^{3,4}$ In the small intestine, angiodysplasia presents a taxing surgical problem, and is usually diagnosed only by arteriography after extensive investigation for unexplained gastrointestinal bleeding. When angiodysplasias are in the small bowel they are usually inaccessible to endoscopists except at laparotomy, when a sterilized flexible colonoscope may be passed orally and manipulated by hand into the small bowel, past the ligament of Treitz. ${ }^{5}$ Forde has examined the small bowel by passing the colonoscope per rectum. ${ }^{6}$ Endoscopy may be preoperative, when vision may be obscured by clots or blood, or intraoperative, ${ }^{7,8}$ when lavage may rapidly clear the intestine and permit accurate identification of a bleeding lesion. The small bowel however, presents special problems of its own. For the surgeon, the main technical problem is that even when identified by angiography, lesions are notoriously difficult to locate at laparotomy, being frequently impalpable, and invisible to the naked eye, ${ }^{1,9,10}$ unless they are actively bleeding at the time of surgery. This dilemma has prompted authors to advocate intraoperative arteriography, either by the transfemoral route, ${ }^{11}$ or by selectively cannulating the suspect mesenteric arcade vessels with a butterfly needle. ${ }^{3,9,10}$ These methods are not without danger; occlusion of the superior mesenteric artery being reported after manipulation of the transfemoral catheter, ${ }^{10,11}$ and necrosis of the bowel wall can result from excessive injection of contrast medium. ${ }^{9}$ Meticulous examination of the small bowel may fail to locate the lesion, despite transillumination with the theatre

Correspondence: S.D. Scott, F.R.C.S

Accepted: 2 June 1987 light. ${ }^{1,9,11}$ Endoscopy is similarly often unfruitful because the majority of lesions are submucosal, ${ }^{1,11}$ and rarely exceed a few millimetres in diameter. ${ }^{13} \mathrm{We}$ describe a simple intraoperative technique used successfully to identify an angiodysplasia in the jejunum, prior to small bowel resection.

\section{Case report}

A 75 year old woman was referred for angiography after extensive negative investigation of recurrent melaena, her haemoglobin having fallen recently to $5.2 \mathrm{~g} / \mathrm{dl}$. Recent gastroscopy, colonoscopy, and barium studies had all proved normal. A vagotomy and pyloroplasty had been performed 3 years ago for a presumed duodenal ulcer, despite which her faecal occult blood tests remained positive. A transfemoral selective superior mesenteric arteriogram showed a hamartomatous leash of vessels in the upper jejunum, with characteristic early venous filling (Figure 1). She was transfused and referred for surgery. At laparotomy the small bowel was meticulously inspected from its serosal surface but no visible vascular lesion could be identified, and there was no palpable abnormality. Transillumination using the theatre light similarly failed to show any abnormality in either the mesentery or the small bowel. A gastroscope (Olympus P3) was then introduced through a jejunostomy, and two soft non-crushing clamps applied to enable a long segment of small bowel to be inflated for inspection. Thorough endoscopic examination of the mucosal surface appeared normal; there was no intraluminal bleeding and no obvious angiodysplasia to be seen. However, by dimming the theatre lights, the endoscope was then noted to be brilliantly transilluminating the distended small bowel from within. The vessels on the wall of the jejunum were perfectly 


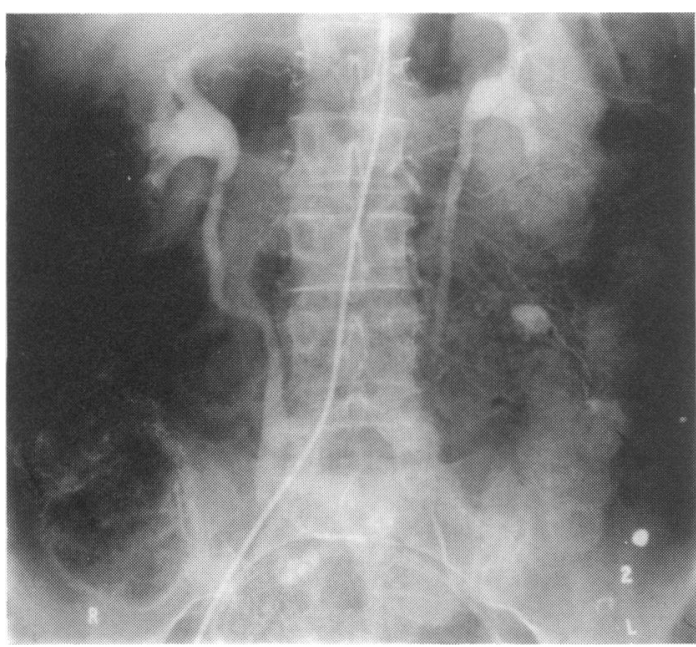

Figure 1 Selective mesenteric angiogram, showing early venous filling (arrowed).

silhouetted as they ran across the muscle layer. All the vessels looked remarkably uniform, except in one area where an extremely tortuous vessel was clearly seen to be macroscopically totally distinct from all the others (Figure 2). This corresponded to the presumed site of the lesion revealed by arteriogram, by counting the branches of the superior mesenteric artery to the arcades, shown by theatre backlighting. Having identified the suspect area we were able to perform a limited small bowel resection with confidence that the lesion was included. The lesion was marked with a black silk stitch to aid the pathologist, though as usual in angiodysplasia, histology of the resected specimen showed no significant abnormality.

\section{Discussion}

In the 30 years since Hirschowitz introduced the first clinically useful flexible fibreoptic endoscope, and Marcozzi \& Montori first described intra-operative $\vec{O}$ colonoscopy in $1972,{ }^{15}$ there have been several reports $\overrightarrow{.}$ of intra-operative endoscopy, ${ }^{2,5-8}$ but few of introduc- $\omega$ tion of the endoscope via an enterotomy, and only one $e^{5}$ of use to identify angiodysplasias of the small bowel. 3

If an arteriovenous malformation is actively bleeding it is relatively easy to identify at laparotomy, but in the quiescent phase practically without exception, of such lesions are not detectable by palpation or inspection by the surgeon. ${ }^{10,11}$ This results in many negative of laparotomies and even 'blind' bowel resections. '을 Arteriovenous malformations are predominantly located in the distribution of the ileocolic and right $z$ colic arteries. ${ }^{13,10}$ Intestinal arteriovenous malformations have been demonstrated by $99 \mathrm{~m}$ Tc-pertechnetate scan $^{14}$ but the lesion was presumed preoperatively to be a Meckel's diverticulum, and laparotomy an extensive malformation measuring $\infty$ $130 \mathrm{~cm}$ was found. Alfidi reports one patient wheresa. malformation was confirmed on two separate angiograms, but at laparatomy, despite theatre light

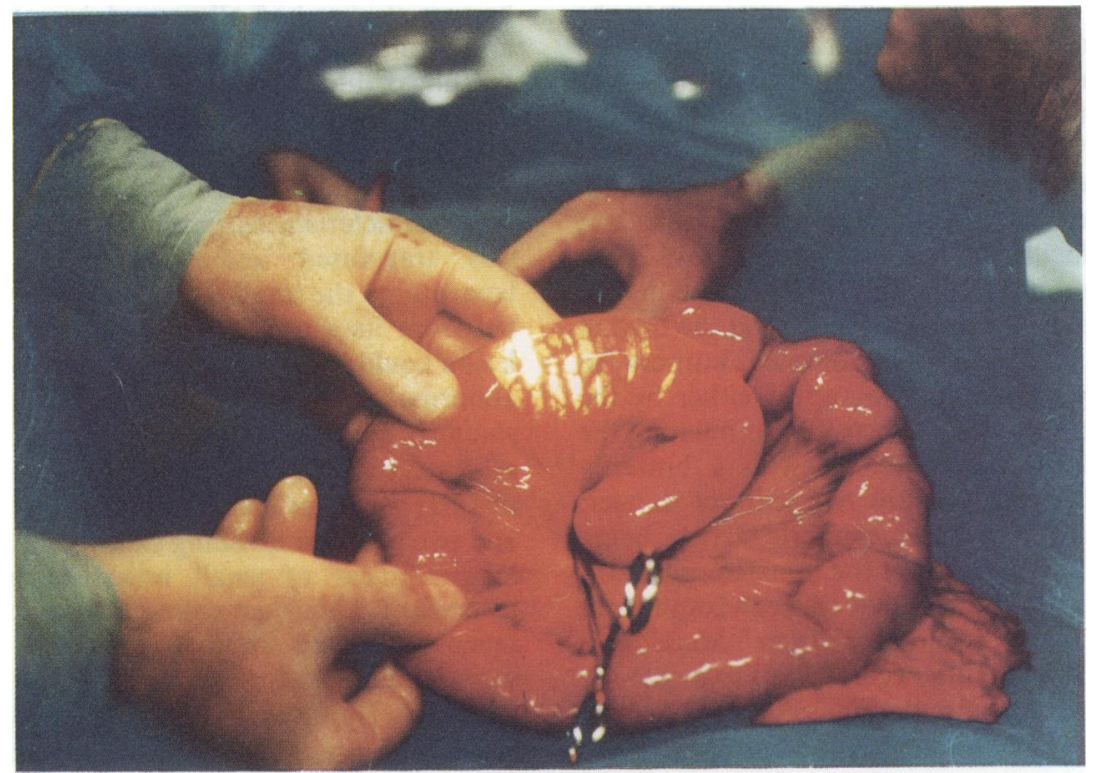


transillumination and endoscopy, the surgeons were unable to find the lesion."

With the bowel in its normal collapsed state, the wall is thick and transillumination with the standard theatre light will display vessels in the mesentery, but not adequately reveal the anatomy of the bowel wall, since two layers of wall are being transilluminated. The advantages of the endoscopic transillumination are that the bowel wall is distended, the light source is relatively brilliant, and only one layer of bowel wall is transilluminated. It is the combination of these three factors which makes this technique so simple and effective.

Transluminal illumination is a very clear technique, and although a better effect is produced by dimming the theatre lights, there is certainly no need to switch

\section{References}

1. Moore, J.D., Thompson, N.W., Appleton, H.D. \& Foley, D. Arteriovenous malformations of the gastrointestinal tract. Arch Surg 1976, 111: 318-319.

2. Williams, C.B. Angiodysplasia. Surgery 1986, 1: 692693.

3. Richardson, J.D., Max, M.H., Flint, L.M. et al. Bleeding vascular malformations of the intestine. Surgery 1978, 84: $430-436$.

4. Boley, S.J. \& Brandt, L.J. Vascular ectasias of colon1986. Dig Dis Sci 1986, 31 (suppl): 265-425.

5. Bowden, T.A., Hocks, V.H. \& Mansberger, A.R. Intraoperative endoscopy, gastrointestinal endoscopy. Ann Surg 1980, 191: 680-687.

6. Forde, K.A. Intraoperative colonoscopy. In Hunt, R.H. \& Waye, J.D. (eds) Colonoscopy, Chapter 11. Chapman and Hall, London, 1981, pp. 189-198.

7. Campbell, W.B., Rhodes, M. \& Kettlewell, M.G.W. Colonoscopy following intraoperative lavage in the management of severe colonic bleeding. Ann R Coll Surg Engl 1985, 67: 290-292.

8. Martin, P.J. \& Forde, K.A. Intraoperative colonoscopy. Dis Colon Rectum 1979, 22: 234-237. them off as suggested by Bowden. ${ }^{5}$

Others have used the technique of intra-operative endoscopy, but passed the endoscope either orally ${ }^{5}$ or rectally ${ }^{6}$ to avoid the risks of enterotomy converting a clean wound into a contaminated wound. Where it is already established that the lesion is an angiodysplasia within the small bowel (by preoperative angiography), a resection is inevitable, and the endoscope can be introduced through an enterotomy without additional risk. By this technique we were able to view the whole of the small bowel in only 5 minutes, whereas others have taken 45 minutes. ${ }^{5}$

The technique of intra-operative transluminal illumination using an endoscope allows accurate, rapid localization of an angiodysplasia, and permits precise definitive surgery with confidence.

9. Margulis, A.R., Heinbecker, P. \& Bernard, H.R. Operative mesenteric arteriography in the search for the site of bleeding in unexplained gastrointestinal haemorrhage. Surgery 1960, 48: 534-539.

10. Crichlow, P.W., Mosenthal, W.T., Spiegel, P.K. \& Howe, R.K. Arteriovenous malformation of the bowel, an obscure cause of bleeding. Am J Surg 1975, 129: 440 448.

11. Alfidi, R.J., Esselstyn, C.D., Tarar, R. et al. Recognition and angiosurgical detection of arteriovenous malformations of the bowel. Ann Surg 1971, 174: 573-582.

12. Robertson, H.D. \& Garhright, J.B. The technique of intraoperative segmental artery arteriography to localise vascular ectasias. Dis Colon Rectum 1985, 28: 71-72.

13. Anon. Angiodysplasia. Lancet 1981, ii: 1086-1087.

14. Wesselhoeft, C.W., Deluca, F.G. \& Luke, M. Positive $99 \mathrm{~m}$ Tc-pertechnetate scan in a child with intestinal arteriovenous malformation. J Pediatr Surg 1986, 21: 71-72.

15. Marcozzi, G. \& Mantori, A. Endoscopy during operation: new diagnostic possibilities. Chir Gastroenterol 1972, 6: $23-25$. 